\title{
Inborn Errors of Metabolism Associated With Autism Spectrum Disorders: Approaches to Intervention
}

\author{
Tamara Žigman', Danijela Petković Ramadža ${ }^{1}$, Goran Šimićn ${ }^{2 *}$ and Ivo Barić1* \\ ${ }^{1}$ Department of Paediatrics, University Hospital Center Zagreb and University of Zagreb School of Medicine, Zagreb, \\ Croatia, ${ }^{2}$ Department of Neuroscience, Croatian Institute for Brain Research, University of Zagreb School of Medicine, \\ Zagreb, Croatia
}

\section{OPEN ACCESS}

Edited by:

Joana M. Gaspar, Federal University of Santa Catarina,

Brazil

\section{Reviewed by: \\ William Griffiths, \\ Swansea University, United Kingdom}

Khue Vu Nguyen,

University of California, San Diego,

United States

${ }^{*}$ Correspondence:

Goran Šimic

gsimic@hiim.hr

Ivo Barić

ivo.baric@kbc-zagreb.hr

Specialty section:

This article was submitted to

Neuropharmacology,

a section of the journal

Frontiers in Neuroscience

Received: 27 February 2021

Accepted: 03 May 2021

Published: 28 May 2021

Citation:

Žigman T, Petković Ramadža $D$,

Šimić G and Barić I (2021) Inborn

Errors of Metabolism Associated With

Autism Spectrum Disorders:

Approaches to Intervention.

Front. Neurosci. 15:673600.

doi: 10.3389/fnins.2021.673600
Increasing evidence suggests that the autism spectrum disorder (ASD) may be associated with inborn errors of metabolism, such as disorders of amino acid metabolism and transport [phenylketonuria, homocystinuria, S-adenosylhomocysteine hydrolase deficiency, branched-chain $\alpha$-keto acid dehydrogenase kinase deficiency, urea cycle disorders (UCD), Hartnup disease], organic acidurias (propionic aciduria, L-2 hydroxyglutaric aciduria), cholesterol biosynthesis defects (Smith-LemliOpitz syndrome), mitochondrial disorders (mitochondrial encephalomyopathy, lactic acidosis, and stroke-like episodes-MELAS syndrome), neurotransmitter disorders (succinic semialdehyde dehydrogenase deficiency), disorders of purine metabolism [adenylosuccinate lyase (ADSL) deficiency, Lesch-Nyhan syndrome], cerebral creatine deficiency syndromes (CCDSs), disorders of folate transport and metabolism (cerebral folate deficiency, methylenetetrahydrofolate reductase deficiency), Iysosomal storage disorders [Sanfilippo syndrome, neuronal ceroid lipofuscinoses (NCL), NiemannPick disease type C], cerebrotendinous xanthomatosis (CTX), disorders of copper metabolism (Wilson disease), disorders of haem biosynthesis [acute intermittent porphyria (AIP)] and brain iron accumulation diseases. In this review, we briefly describe etiology, clinical presentation, and therapeutic principles, if they exist, for these conditions. Additionally, we suggest the primary and elective laboratory work-up for their successful early diagnosis.

Keywords: autism spectrum disorder, early diagnosis, genetic testing, inborn errors of metabolism, therapeutic principles

\section{INTRODUCTION}

Autism spectrum disorder (ASD) is a behavioral developmental disorder defined by the impairment of communication and social interaction. It usually starts before age three and children with autism can be recognized by stereotyped and repetitive patterns of behavior as well as their restricted activities and interests. ASD varies in degrees of severity, occurring in $1 \%$ of the population, and its prevalence has been increasing during the last three decades (Maenner et al., 2020). The causes of different subtypes of autism lay in the complex landscape of environmental, genetic 
and epigenetic influences (Waye and Cheng, 2018; Šimić et al., 2020). Although the etiology of most cases of ASD is still unknown, numerous studies have shown that there is a strong heritable component. Change (mutation or premutation) in the FMR1 gene sequence on the chromosome $\mathrm{X}$ that results in fragile X syndrome (FXS) or fragile X-premutation tremor/ataxia syndrome (FXTAS) are among the leading genetic causes of ASD (Šarac et al., 2011; Ghaziuddin and Al-Owain, 2013). Current genetic testing strategies, including the combination of molecular cariotypisation and exome sequencing, are the most successful diagnostic approaches, yielding exact diagnosis in about $20-25 \%$ of the cases. The term "non-syndromic" autism refers to patients without dysmorphic characteristics or any other disease or additional signs or symptoms than those known to be associated with ASD. On the other hand, the term "syndromic" is being used to describe individuals with clinical characteristics additional to ASD, such as epilepsy, motor deficits, developmental delay and regression, dysmorphic features, and other manifestations or diseases that can contribute to autistic phenotype (Campistol et al., 2016).

It has been recently proposed that a significant number of ASD cases could be associated with various metabolic abnormalities, some of them identifiable only through untargeted metabolomic profiling, simultaneously opening additional space for therapeutic attempts (Glinton and Elsea, 2019).

\section{Inborn Errors of Metabolism}

Clinical symptoms deriving from central nervous system (CNS) occur in more than $50 \%$ of patients with inborn errors of metabolism (IEM). In addition to neurological or somatic manifestations, psychiatric symptoms are also the presenting sign in $2-5 \%$ of IEM cases (Saudubray and García-Cazorla, 2018). Recent reports emphasized the causal role of IEMs in ASD, meaning that some IEM could be prevented, especially in population with high level of consanguinity (Celestino-Soper et al., 2012; Novarino et al., 2012). Many IEM are treatable conditions; in some of them positive effects are observed when treatments were started early in life. Phenylketonuria (PKU), the prototype of IEM successfully diagnosed by neonatal screening program and treated with diet if initiated early in life, is characterized by intellectual disability and autism (Asato et al., 2015). Possible IEM should be considered in the diagnostic approach of patients with syndromic ASD because for many of them there is a chance for intervention and improvement (Márquez-Caraveo et al., 2020). Spilioti et al. (2013) found significant proportion of patients with an IEM in patients with autism. For most of the IEM, ASD is not a single symptom, but accompanied with other neuropsychiatric and somatic disturbances (Simons et al., 2017). The data of Schiff and colleagues provide a large cohort of non-syndromic autistic subjects for whom a systematic metabolic work-up has been carried-out (Schiff et al., 2011). Their data suggest that usual metabolic workup should not be done routinely in nonsyndromic ASD because the prevalence of IEM in the group of non-syndromic ASD is not higher than in general population $(<0.5 \%)$. In the case of non-syndromic ASD, a precise clinical workup represents a good clinical practice (Schiff et al., 2011). In this review, we give an overview of IEM characterized by symptoms of ASD, propose diagnostic approach to assess such cases in clinical practice, and suggest possible specific therapies.

\section{ASD ASSOCIATED WITH INBORN ERRORS OF METABOLISM}

\section{Disorders of Amino Acid Metabolism Phenylketonuria}

Phenylketonuria (PKU) is the most common IEM in the group of aminoacidopathies. It is caused by biallelic mutations in the phenylalanine hydroxylase (PAH) gene with consequent lowering of PAH activity. PAH metabolizes phenylalanine to tyrosine, a process which requires the cofactor tetrahydrobiopterin (BH4). Deficient activity of PAH results in elevated concentration of phenylalanine in the blood and toxic levels in the CNS. If untreated, PKU is accompanied with severe and progressive intellectual disability, but may also be associated with symptoms such as autism, seizures, and motor deficits. Psychiatric or psychological difficulties may become apparent if the patient is not compliant with the diet (Blau et al., 2010). Newborn screening programs successfully diagnose patients with PKU. Treatment with a low phenylalanine diet in the postneonatal period has resulted in a worldwide population of over 50 thousand PKU individuals without cognitive deficits (ten Hoedt et al., 2011). $\mathrm{BH} 4$, the natural cofactor of $\mathrm{PAH}$, may be effective in about $50 \%$ of PKU patients who are BH4-responsive to increase phenylalanine tolerance (van Wegberg et al., 2020). Possible new treatment options include enzyme therapy with phenylalanine ammonia lyase, enzyme that metabolizes phenylalanine, and gene therapy (van Wegberg et al., 2017).

\section{Homocystinuria (Cystathionine $\beta$-Synthase Deficiency)}

Cystathionine $\beta$-synthase (CBS) deficiency, also known as classical homocystinuria (HCU), is caused by biallelic mutations in the CBS gene. CBS deficiency prevents the conversion of homocysteine (Hcy) to cystathionine, resulting in homocysteine increase. Subjects with classical homocystinuria may have a clinical picture of varying severity and with different age of onset. It can start in childhood as a severe multisystemic disease or stay asymptomatic until adulthood (Morris et al., 2017). Untreated patients may have various symptoms, including osteoporosis, thromboembolic events, and intraocular lens dislocation. These patients can also have a variety of CNS disturbances, including symptoms of ASD (Abbott et al., 1987; Kiykim et al., 2016). Extrapyramidal signs and seizures are frequent features too (Morris et al., 2017). Treatment options include vitamins $B_{6}$, $\mathrm{B}_{12}$ and folate supplementation, low-methionine diet and betaine (Kruger, 2017). Current efforts for developing novel therapies for HCU include enzyme replacement therapy with recombinant enzyme OT-58 that has been shown effective in lowering plasma and tissue homocysteine, ameliorating metabolic balance and clinical symptoms in a phase II clinical study (clinical trial 
NCT03406611). Gene therapy using adenovirus or minicircle DNA is also being appraised (Bublil and Majtan, 2020).

\section{S-Adenosylhomocysteine Hydrolase Deficiency}

S-adenosylhomocysteine hydrolase deficiency (SAHHD) is a rare disorder of methionine metabolism characterized by (not constant) hypermethioninemia, elevation of S-adenosylmethionine (AdoMet) and S-adenosylhomocysteine (AdoHcy, Barić et al., 2004). AdoHcy is an inhibitor of different methytransferases, enzymes that transfer methyl group to various molecules such as DNA, RNA, lipids, proteins, amino acids, and others. The removal of adenosine and Hcy under physiological conditions is sufficient to direct the flux toward hydrolysis. Hydrolysis of AdoHcy plays a critically important role in the regulation of reactions of biological methylation processes (Barić et al., 2004).

Clinical presentations vary from severe perinatal to milder forms and include various combinations of myopathy with elevated creatine kinase (hyperCKemia), behavioral disturbances, developmental delay, dysmyelination, coagulopathy, strabismus, and hepatic disease. Diet with low methionine intake can decrease and sometimes normalize plasma AdoMet and AdoHcy concentrations, with positive effects on methylation and clinicobiochemical parameters. Together with low protein/methionine diet, creatine and phosphatidylcholine supplementations have been used in some individuals, although without firm evidence of clinical improvement. Myopathy is less responsive to treatment, whereas liver, coagulation and neurological abnormalities are more responsive (Barić et al., 2017). Liver transplantation in a single patient improved cognitive development, especially gross motor, language and social skills (Strauss et al., 2015).

\section{Branched-Chain $\alpha$-Keto Acid Dehydrogenase Kinase Deficiency}

Biallelic mutations in the $B C K D K$ gene that codes for the kinase behind the negative regulation of the branched-chain $\alpha$-keto acid dehydrogenase complex (BCKD) have been associated with clinical symptoms of autism with seizures (Novarino et al., 2012). Normalization of plasma branched-chain amino acids (BCAA) improves hyperactivity, attention span, gross motor and communication skills (Burrage et al., 2014). Supplementation with high-protein diet and frequent BCAA supplement dosing throughout the day are required for normalization of BCAA plasma concentrations (Burrage et al., 2014; García-Cazorla et al., 2014).

\section{Urea Cycle Disorders}

The urea cycle is a metabolic pathway that serves to eliminate excess of nitrogen, arising primarily as ammonia. Nitrogen in small quantities is essential substance for growth and cellular equilibrium, but excessive ammonia can lead to lifethreatening consequences. In its non-ionized form ammonia easily crosses the blood-brain barrier and enters the CNS. In case of a severe metabolic crisis, a great amount of ammonia can accumulate in the blood and tissues, especially in the CNS that is most vulnerable. As the acute hyperammonaemia increases extracellular glutamate and causes excitotoxic cell death it comes as no surprise that neurological disturbancies such as ataxia, tremor, and seizures may ensue (Matsumoto et al., 2019). Urea cycle disorders (UCD) comprise deficiency of one of several enzymatic steps or transporters of the urea cycle. Clinical presentation is characterized by severe hyperammonaemic crisis in the newborn/infancy period, while the late-onset form is primary characterized by neurological symptoms from infancy through adulthood with possibility of metabolic decompensating and hyperammonaemia triggered by catabolic states. Besides other neurological symptoms, ASD was described in patients with carbamoyl phosphate synthetase deficiency, ornithinetranscarbamylase (OTC) deficiency, citrullinaemia type 1, and arginase deficiency (Frye, 2015). It is of immediate urgency to reduce the plasma ammonia concentration when hyperammonaemia develops. Acute management relies on ammonia detoxification by giving ammonia scavengers (sodiumbenzoate or sodium-phenylbutirate), but hemodialysis and hemodiafiltration are the most efficient treatment strategies for plasma ammonia reduction. In parallel, catabolic state is reversed by providing high amounts of intravenous glucose and lipids. Arginine-hydrochloride is given to stimulate urea cycle reactions (except in case of arginase deficiency). Chronic treatment with low-protein diet prevents a catabolic state and recurrence of hyperammonaemia. In severe neonatal forms, prognosis is unfavorable if severe metabolic crisis develops. In late-onset forms improvement of symptoms has been reported with treatment. Liver transplantation can change the unfavorable clinical course in male patients with OTC deficiency (Matsumoto et al., 2019).

\section{Hartnup Disease}

Hartnup disease is a disfunction of the $\mathrm{B}^{0} \mathrm{AT} 1$ protein that results in overexcretion of neutral amino acids in feces and urine (Hashmi and Gupta, 2021). B ${ }^{0} \mathrm{AT} 1$ is a transporter responsible for the absorption of neutral amino acids in small intestine and renal tubules. As tryptophan serves as a precursor for niacin, deficiency of tryptophan and niacin manifests as pellagra-like skin changes and neurological disturbancies (Orbak et al., 2010). Along with ASD, clinical presentation includes reversible and intermittent episodes of neurological and symptoms affecting the skin. Neurological symptoms include ataxia, tremor, depression, mood disorders, epilepsy and psychosis. The skin eruptions are present at areas exposed to sun and have erythematous and scaly appearance. The disease is treatable with high protein diet and nicotinamide (Hashmi and Gupta, 2021).

Loss of CLTRN protein and its functions results in a disorder of similar biochemical phenotype to Hartnup disease. CLTRN gene encodes the protein collectrin, a homolog of angiotensinconverting enzyme 2 (ACE2), which is involved in transportation and activation of $\mathrm{B}^{0} \mathrm{AT} 1$ protein in the renal epithelium (Singer and Camargo, 2011; Pillai et al., 2019).

\section{Organic Acidurias}

\section{Propionic Aciduria}

Propionic aciduria (PA) is an inborn error of branched chain amino acids metabolism, defined by accumulation of propionic acid due to deficiency of enzyme propionyl-CoA 
carboxylase (Baumgartner et al., 2014). Clinical features of PA may occur due to accumulation of toxic metabolites, altered mitochondrial energy metabolism, carnitine depletion, and coenzyme A sequestration. Acute illness may be presented by metabolic acidosis, acute alterations of consciousness or encephalopathy due to hyperammonaemia, anorexia, nausea, and vomiting. Chronic complications include poor growth, movement disorders, progressive spastic quadriparesis, epilepsy, cardiac dysfunction, osteopenia/osteoporosis, and functional immunodeficiency (Fraser and Venditti, 2016). Neurological complications include epilepsy, intellectual disability, and ASD (Ghaziuddin and Al-Owain, 2013). PA is treated with proteinrestricted diet, precursor-free amino acid supplementation, and carnitine supplementation due to secondary carnitine deficiency. Acute treatment aims to stop catabolism and enable ammonia detoxification by giving sodium benzoate, L-arginine, and $\mathrm{N}$-carbamyl-glutamate. Severe metabolic acidosis sometimes requires hemodiafiltration. Despite adequate therapy, significant number of patients have intellectual disability and psychiatric problems, thus requiring psychosocial support from childhood throughout adult life (Baumgartner et al., 2014).

\section{L-2 Hydroxyglutaric Aciduria}

L-2-hydroxyglutaric aciduria is a metabolic disorder affecting CNS and characterized by elevated concentrations of L2-hydroxyglutaric acid in plasma, urine and cerebrospinal fluid (CSF) due to the deficiency of L-2-hydroxyglutarate dehydrogenase. Disease is defined by progressive neurological symptoms: ataxia, mental deterioration, subcortical leukoencephalopathy, and ASD (Zafeiriou et al., 2008). A few successful therapeutic trials with riboflavin and flavin adenine dinucleotide (FAD) have been reported in subjects with L2-hydroxyglutaric aciduria. Yilmaz (2009) described the first patient treated with riboflavin in whom a partial improvement in motor and cognitive performance and significant decrease of urinary excretion of L-2-hydroxyglutarate was observed. Another approach, described by Samuraki et al. (2008) in an adult patient, is based on treatment with levocarnitine and FAD, which resulted in improvement of dystonia and tremor as well as in a significant decrease of urinary excretion of L-2hydroxyglutarate. These examples suggest that supplementation of riboflavin improves the enzymatic activity by raising the intracellular concentration of FAD. Therefore, they may be categorized into a group of vitamin-responsive IEM (Van Schaftingen et al., 2009).

\section{Cholesterol Biosynthesis Defects}

\section{Smith-Lemli-Opitz Syndrome}

Smith-Lemli-Opitz syndrome (SLOS) is an autosomal recessive disorder caused by biallelic mutations in the DHCR7 gene, resulting in impaired biosynthesis of cholesterol. Besides dysmorphic features and intellectual disability, autistic symptoms are often a part of the SLOS behavioral phenotype (Thurm et al., 2016). SLOS is characterized by elevated 7dehydrocholesterol and low plasma cholesterol levels. Some of the major consequences of cholesterol deficiency include abnormal formation of the CNS, face and limbs via an effect on sonic hedgehog $(\mathrm{SHH})$ signaling pathways during development (Li et al., 2020) and its effect on steroidogenesis, particularly that of dihydrotestosterone. Therapeutic intervention with supplementation of cholesterol and symptomatic treatment after early diagnosis decreases mortality and improves longterm outcome (Donoghue et al., 2018). It is also important to know that several common antipsychotic, antidepressant, and anxiolytic compounds, including aripiprazole, buspirone, fluoxetine, haloperidol, nefazodone, perospirone, and trazodone may induce an in vitro biochemical profile similar to SLOS (Kim et al., 2016).

\section{Mitochondrial Disorders of Energy Production}

Mitochondrial dysfunction is a common metabolic disturbance observed in ASD subjects. At the same time, different clinical features and biochemical abnormalities seen in ASD subjects can be linked to mitochondrial dysfunction (Frye and Rossignol, 2011). Although it seems that mitochondrial dysfunction can unify the seemingly disparate abnormalities associated with ASD, mitochondrial failure is commonly a secondary phenomenon related to other biological processes, such as chronic immune dysfunctions and increased oxidative stress that have been observed in ASD patients (James et al., 2009; Li et al., 2009; Malik et al., 2011).

Mitochondrial encephalomyopathy, lactic acidosis, and stroke-like episodes (MELAS) is one of the most frequent mitochondrial disorders, inherited maternally. Clinical criteria include: (1) stroke-like episodes before age of 40, (2) encephalopathy with seizures and/or cognitive deficits, and (3) mitochondrial myopathy accompanied by lactic acidosis and/or ragged-red fibers (El-Hattab et al., 2015). In addition to its neurological manifestations, MELAS syndrome can include a clinical picture of ASD (Ahmadabadi et al., 2020). Connolly and collaborators described kindred with MELAS, autism, cardiomyopathy, and rhabdomyolysis associated with the A3260G mtDNA mutation (Connolly et al., 2010). Treatment of MELAS syndrome with L-arginine, coenzyme Q, creatine monohydrate, and carnitine can be beneficial. Valproic acid, metformin, and dichloroacetate should be avoided (El-Hattab et al., 2015).

\section{Neurotransmitter Disorders \\ Succinic Semialdehyde Dehydrogenase Deficiency}

Succinic semialdehyde dehydrogenase deficiency (SSADH-D) is a genetic disease caused by disrupted metabolism of the $\gamma$-amino butyric acid (GABA). Impaired activity of the mitochondrial enzyme SSAD leads to the accumulation of $\gamma$-hydroxybutyric acid (GHB). The SSADH-D has highly heterogeneous clinical picture, with varying degrees of autism, mental retardation, hypotonia, ataxia, epilepsy, and a delay in speech development. There has been no approved curative therapy for the disease yet, but vigabatrin can be beneficial by reducing accumulation of SSA and GHB through inhibition of GABA transaminase. Inhibition with vigabatrin, however, has no effect on peripheral GABA transaminase, so the 
GHB keeps rising and reaches the CNS (Pearl et al., 2016). Many therapeutic approaches are currently being studied (Didiášová et al., 2020).

\section{Disorders of Purine Metabolism Adenylosuccinate Lyase Deficiency}

Adenylosuccinate lyase (ADSL) deficiency is a disorder of purine metabolism that reduces purine de novo synthesis and purine nucleotide recycling. ADSL was first described in Jaeken and Van den Berghe (1984), who discovered succinylpurines in the plasma, urine, and CSF of a few patients with severe psychomotor delay and autism. Growth and development might be normal in the newborn period (Jurecka et al., 2015), but in severe forms neurological symptoms might be evident immediately after birth (Ciardo et al., 2001). They include acute and chronic encephalopathy, behavioral abnormalities, and ASD. Treatment relies on epilepsy treatment. Intervention with D-ribose administration, which increases levels of phosporibosylpyrophosphate and stimulates purine synthesis de novo, has only limited success (Jurecka et al., 2015).

\section{Lesch-Nyhan Syndrome}

Lesch-Nyhan syndrome (LNS) is an X-linked disorder caused by mutations in the HPRT1 gene, coding for hypoxanthineguanine phosphoribosyltransferase (HPRT). Symptoms include dystonia, intellectual disability, gout, autism, and behavioral changes such as self-mutilation. Pathogenesis is unclear and it remains to be uncovered how deficits in hypoxanthine and guanine recycling lead to a severe phenotype. Upon identification of several isoforms of amyloid precursor protein $(A P P)$ mRNA with a deletion followed by an insertion that accounted for epigenetic control of genomic rearrangements of $A P P$ gene in fibroblasts of patients with LNS, Nguyen proposed a role of epistasis between mutated HPRT1 and APP genes affecting the regulation of alternative $A P P$ pre-mRNA splicing as a possible pathophysiological mechanism of the severe neurobehavioral phenotype of LNS (Nguyen, 2019). Patients excrete large amounts of uric acid. Allopurinol prevents urate nephropathy. Treatment with allopurinol has no effect on neurological symptoms (Bell et al., 2016).

\section{Cerebral Creatine Deficiency Syndromes}

Creatine serves as a donor of high-energy phosphates for the synthesis of hydrolyzed adenosine triphosphate. The role of creatine transporter coded by the SLC6A8 gene on chromosome $\mathrm{X}$ is to translocate creatine across the cell membrane in the cytoplasm of neurons and myocytes. Mutations in the $S L C 6 A 8$ gene lead to cerebral creatine deficiency syndrome (CCDS) with a spectrum of clinical manifestations, including severe intellectual delay, autism, epilepsy, and motor dysfunction. Females heterozygous for the $S L C 6 A 8$ pathogenic mutation are asymptomatic or have mild intellectual delay. The other two CCDS disorders are caused by the deficiencies of two enzymes involved in the synthesis of creatine: arginine-glycine amidinotransferase (AGAT) and guanidinoacetate methyltransferase (GAMT).
All three conditions result in creatine depletion in the brain (Farr et al., 2020). GAMT and AGAT are treated with oral creatine monohydrate to restore cerebral creatine levels. Therapy of GAMT using ornithine supplementation and protein dietary restriction has only limited success (Mercimek-Andrews and Salomons, 2015).

\section{Disorders of Folate Transport and Metabolism Cerebral Folate Deficiency}

Cerebral folate deficiency (CFD) is any neurological condition with a low CSF concentration of 5-methyltetrahydrofolate (5-MTHF) and normal folate plasma concentrations. This syndrome is characterized by a wide variety of clinical symptoms, from irritability and sleep disturbances to severe seizures, developmental regression, autism, progressive ataxia and extrapyramidal symptoms, including choreoatethosis (Hyland et al., 2010). CFD is a treatable condition if timely recognition is followed by treatment with folinic acid perorally. Treatment with folic acid is contraindicated as it may exacerbate the 5 -MTHF deficiency in the CNS. Autoimmunity against the folate receptor (transporter of 5-MTHF into the CSF) and mutations in the folate receptor 1 (FOLR1) gene are the primary causes of CFD. Secondary CFD has been observed in other IEM, including mitochondrial diseases, serine deficiency, and pyridoxine-dependent epilepsy (Pope et al., 2019).

\section{Methylenetetrahydrofolate Reductase Deficiency}

Methylenetetrahydrofolate reductase deficiency (MTHFR-D) is the most common genetic cause of hyperhomocysteinemia. MTHFR has an important role in the conversion of Hcy to methionine. A common genetic variant in the MTHFR gene is the c.677C > T polymorphism (rs1801133), where the common variant is less active at higher temperatures (Dean, 2012). In comparison to controls, subjects with two copies of this variant usually have higher Hcy levels and lower serum folate levels ("TT homozygous"). Another common MTHFR variant, c.1298A > C, is not associated with homocysteinemia alone, but only combined heterozygosity of c.1298A $>\mathrm{C}$ and $\mathrm{c} .677 \mathrm{C}>\mathrm{T}$ results in an outcome similar to TT genotype. Pathogenic variants in the MTHFR gene can cause an autosomal recessive IEM with high concentrations of Hcy in plasma and urine. This, in turn, may cause developmental delay, thrombosis, eye disorders, and osteoporosis (Dean, 2012). Importantly, the TT genotype is frequent among ASD patients and their mothers, compared to the general population. The results of the study of Sadigurschi and Golan (2019) support the view that the maternal MTHFR genotype is associated with increased risk for ASD in children. The study of Orenbuch et al. (2019) showed that prenatal nutritional intervention by adding folic acid and choline in the diet of pregnant MTHFR-deficient mice reduce autistic-like behaviors among offspring that are MTHFR-deficient. Further studies are needed to better define the role of methionine cycle in the pathogenesis of ASD. 


\section{Lysosomal Storage Disorders}

\section{Muccopolysaccharidosis Type III (Sanfilippo Syndrome)}

Mucopolysaccharidosis type III (MPS III) is a multisystemic lysosomal storage disease characterized by progressive neurodegeneration characterized by severe developmental regression, intellectual disability, autism, and other behavioral problems. Multisystemic manifestations include hearing loss, musculoskeletal problems (contractures, joint stiffness, hip dysplasia and scoliosis), respiratory tract infections, and valvular cardiac disease (Wagner and Northrup, 2019). Patients are commonly misdiagnosed as having idiopathic developmental delay, attention deficit/hyperactivity disorder or ASD with unnecessary testing procedures and treatment attempts. Neurological decline is inevitable (Wijburg et al., 2013). Treatment is symptomatic with therapies such as enzyme replacement; however, the latest developments could push gene therapy into the mainstream (Seker Yilmaz et al., 2021).

\section{Neuronal Ceroid Lipofuscinoses}

Neuronal ceroid lipofuscinoses (NCL) are a group of autosomal recessive, progressive lysosomal storage disorders with dominantly neurological symptomatology. This group of diseases has a high genetic heterogeneity and variable clinical presentation. The most common forms are infantile NCL (INCL), late infantile NCL (LINCL), and juvenile NCL (JNCL). Common symptoms include epilepsy, developmental regression, and ophthalmologic abnormalities. Madaan et al. (2020) described a patient with autistic regression and epilepsy, along with EEG photosensitivity that is considered as an early sign of Batten disease. Cerliponase $\alpha$, a recombinant human tripeptidyl peptidase 1 enzyme replacement therapy, became the first globally approved treatment for LINCL_Batten disease in 2017, signifying major therapeutic progress (Johnson et al., 2019).

\section{Niemann-Pick Disease Type C}

Niemann-Pick disease type $\mathrm{C}$ is an autosomal recessive disorder caused by mutations in NPC1 (in 95\% of cases) or NPC2 (in about $5 \%$ of cases) genes. When either of two proteins, NPC1 or NPC2, is not functional, it leads to impaired cellular trafficking of endocytosed LDL cholesterol and its accumulation in the lysosomes. The clinical presentation is highly heterogeneous, with an age of onset ranging from the perinatal period to late adulthood. Visceral signs such as fetal hydrops, hepatosplenomegaly, and cholestatic jaundice in early forms usually preceed neurological and psychiatric manifestations. Manifestations of neurological forms (lateinfantile and juvenile forms) are ataxia, clumsiness, handwriting difficulties, impaired attention, vertical supranuclear gaze palsy and gelastic cataplexy. Some patients develop seizures. Compared to younger-onset patients, individuals with the adolescent/adult onset more frequently develop psychiatric symptoms and cognitive impairment (Newton et al., 2018).

Oxysterols (cholestane-3 $\beta, 5 \alpha$ and $6 \beta$-triol and 7-ketocholesterol are elevated in most patients, but they can also be elevated in the deficiency of lysosomal acid lipase and in neonatal cholestasis in general. Gene analysis is diagnostic.
Miglustat is currently the only causal treatment for neurological manifestations (Geberhiwot et al., 2018; Patterson et al., 2020).

\section{Cerebrotendinous Xanthomatosis}

Cerebrotendinous xanthomatosis (CTX) is a disorder of bile acid synthesis caused by mutations in the cytochrome P450 CYP27A1 gene. These mutations cause dysfunction of sterol 27-hydroxylase enzyme, resulting in incomplete oxidation of the cholesterol side chain. Although CTX does not belong to the lysosomal diseases, by its clicial presentation it resembles storage diseases. Hence, it is often classified in this group. CTX is characterized by high levels of plasma cholestanol and accumulation of cholestanol and cholesterol in the CNS and tendons, which is manifested as tendon xanthomas. Typical clinical symptoms of CTX include chronic diarrhea, tendon xanthomas, bone fractures, bilateral cataracts, and neurological dysfunction. The disease is progressive, particularly due to severe neurological presentations that may include autism, intellectual disability, psychiatric and behavioral problems. Treatment with chenodeoxycholic acid is the current standard of care; if initiated early, it can stop the progression of the disease (Duell et al., 2018). At present, level of $7 \alpha$-hydroxycholestenone appears to be the best biochemical marker for the evaluation of CTX treatment (Lütjohann et al., 2020).

Although Smith-Lemli-Opitz syndrome, Niemann-Pick disease type C and CTX all affect cholesterol metabolism and can present with psychiatric symptoms including autistic features, other clinical signs, metabolic background and treatment approaches do not overlap.

\section{Disorders of Copper Metabolism Wilson Disease}

The key clinical features of Wilson disease include acute episodes of hemolysis in association with acute liver failure, cirrhosis, neuropsychiatric disturbances including autistic features, and eye manifestations such as Kayser-Fleischer ring. Psychiatric and behavioral symptoms are common and may precede other neurological or hepatic signs and symptoms. Wilson disease is a disorder of copper metabolism, caused by biallelic mutations in the $A T P 7 B$ gene, and characterized by low serum ceruloplasmin levels and elevated daily urinary copper excretion (Ferenci, 2017). Current treatment includes avoiding copper-rich foods, reducing copper absorption with zinc, chelation therapy to remove copper from tissues, and symptomatic treatment (Mulligan and Bronstein, 2020). Early recognition is prerequisite for treatment success (Mulligan and Bronstein, 2020).

\section{Disorders of Haem Biosynthesis \\ Acute Intermittent Porphyria}

Mutations in hydroxymethylbilane synthase gene (HMBS) cause autosomal dominant acute intermittent porphyria (AIP). AIP is characterized by life-threatening neurovisceral attacks and increased risk of hepatocellular carcinoma, hypertension, and kidney failure. The only possible treatment is liver transplantation (Bustad et al., 2020). Luder et al. (2009) described a 15-year-old girl with AIP, whose first symptoms were autistic 
features at the age of 4 . Visceral symptoms have occurred in the later course and the diagnosis of AIP was confirmed by the finding of a known causative AIP mutation (Luder et al., 2009).

A new therapeutic approach to AIP is RNA interference-based therapy (Givosiran), which decreases aminolevulinic acid syntase mRNA levels. Another promising approach is gene therapy that targets hepatocytes harboring mutated porphobilinogen deaminase (PBGD) gene (Spiritos et al., 2019).

\section{Brain Iron Accumulation Diseases}

Neurodegeneration with brain iron accumulation (NBIA) is a group of genetic diseases characterized by movement disorders such as parkinsonism and dystonia, psychomotor delay, and early death. There is still no established therapy available to mitigate or stop the progression of these diseases (Di Meo and Tiranti, 2018). Two of these diseases are characterized by autistic regression as a dominant clinical symptom: panthotenate kinase-associated neurodegeneration (PKAN) and $\beta$-propeller protein-associated neurodegeneration (BPAN). Veeravigrom and colleagues described a girl diagnosed with ASD at the age of three, later confirmed to have PKAN. The brain MRI at 15 year of age showed abnormally low T2 signal intensity in the globus pallidus and posterior limb of internal capsule bilaterally due to iron accumulation ("eye of the tiger" sign), as well as cortical atrophy (Veeravigrom et al., 2014). Yogananthan and colleagues described a girl with seizures, autistic regression, intracranial calcification, and iron accumulation in the nucleus niger and globus pallidus. The diagnosis of BPAN was established by identification of pathogenic variant in WD repeat domain 45 (WDR45) gene encoding for $\beta$ propeller protein. Current treatment is only palliative (Yoganathan et al., 2016).

\section{DIAGNOSTIC AND THERAPEUTICAL APPROACHES}

Many IEM present with syndromic ASD, most commonly as an associated symptom. Diagnostic approach includes wide neuroradiologic, metabolic, and genetic workup (Polšek et al., 2011). In this review, we briefly describe etiology, clinical presentation, and therapeutic principles, if available, for several groups of IEM in differential diagnosis of ASD. Many of them are nowadays diagnosed and successfully prevented by neonatal screening program. Based on the list of potentially treatable IEM mentioned in this paper, we suggest following laboratory work-up: ammonia and lactate concentration, acid-base balance, plasma amino acid analysis, urinary organic acid analysis, urinary amino acids, plasma or dry blood spot acylcarnitine profile, total cholesterol, total homocysteine, vitamin $\mathrm{B}_{12}$ concentration, and uric acid. Further, more specific laboratory tests such as purine and pyrimidine analysis, CSF analysis (amino acids, folic acid concentration), cholestanol concentration, urinary glycosaminoglycans and porphyrins, copper excretion, and ceruloplasmin concentration should be done based on additional leading symptoms or laboratory results, but should not be a part of the primary workup. All laboratory tests should be done after a detailed anamnesis and clinical examination with special attention on associated neurological abnormalities. Brain MRI with spectroscopy and electroencephalogram have their place in the primary workup of an ASD patient due to several specific findings that can be leading to a proper diagnosis (CCDSs, NBIA). Here, we do not focus on common genetic causes of ASD such as FXS, tuberous sclerosis, Rett syndrome or on rare dysmorphic syndromes and genetic diagnostic tests such as molecular karyotyping (copy number variants could be found in $10-15 \%$ of cases), PCR assay or single specific gene analyses. In the end, unspecific genomic approaches like whole exome sequencing could be useful in diagnosing rare disorders missed by proposed diagnostic workup and will reveal the etiology in about $30-40 \%$ of syndromic ASD cases (Schaefer et al., 2013).

Over the years, ample evidence has accumulated to suggest that children with ASD have different biochemical profiles compared to healthy children. For example, ageindependent hyperserotonemia is present in approximately one-third to one-half of subjects with ASD (Šimić et al., 2020). Untargeted metabolomics, although still not implemented in routine clinical practice, has been shown to be a promising approach in screening of underlying biochemical abnormalities, discovering new specific biomarkers and directing treatment (Glinton and Elsea, 2019).

An interesting study was done in Greece where a large cohort of children with confirmed ASD were screened for the presence of IEM (Spilioti et al., 2013). Their data provide the evidence for a new potential biomarker-3-hydroxyisovaleric acid as well as a few novel treatment approaches for children with ASD: biotin supplementation and a ketogenic diet elicited mild to significant improvement in clinical picture (Spilioti et al., 2013).

Basic six therapeutic principles of IEM include: (1) substrate reduction therapy (as in PKU), (2) removal of toxic metabolites (e.g., urea cycle disorders), (3) product supplementation (e.g., cerebral creatine deficiency disorders), (4) stimulating of deficient enzyme activity (e.g., $B_{6}$ supplementation in CBS deficiency), (5) enzyme replacement therapy (e.g., in LINCL) or 6) organ transplantation (in some cases of UCD and AIP). As exemplified in parentheses, these principles can be also observed throughout the IEM listed above.

\section{Methionine Cycle and its Disturbances as Well as Folic Acid Enigma Deserve Special Attention Regarding Therapeutic Trials and Opinions}

Periconceptional folic acid supplementation in pregnant women along with the dietary and blood folate concentrations in children with ASD have been shown as environmental factors contributing to the incidence of autism. Moreover, children with autism have hyperhomocysteinemia compared to controls, whereas serum levels of folate and vitamin $\mathrm{B}_{12}$ may be diminished or normal. This can be due to lower folate intake in these subjects. Furthermore, folinic acid supplementation in ASD subjects with low 5-MTHF levels in CSF leads to the normalization of folate levels and alleviates some symptoms of ASD (Castro et al., 2016). 


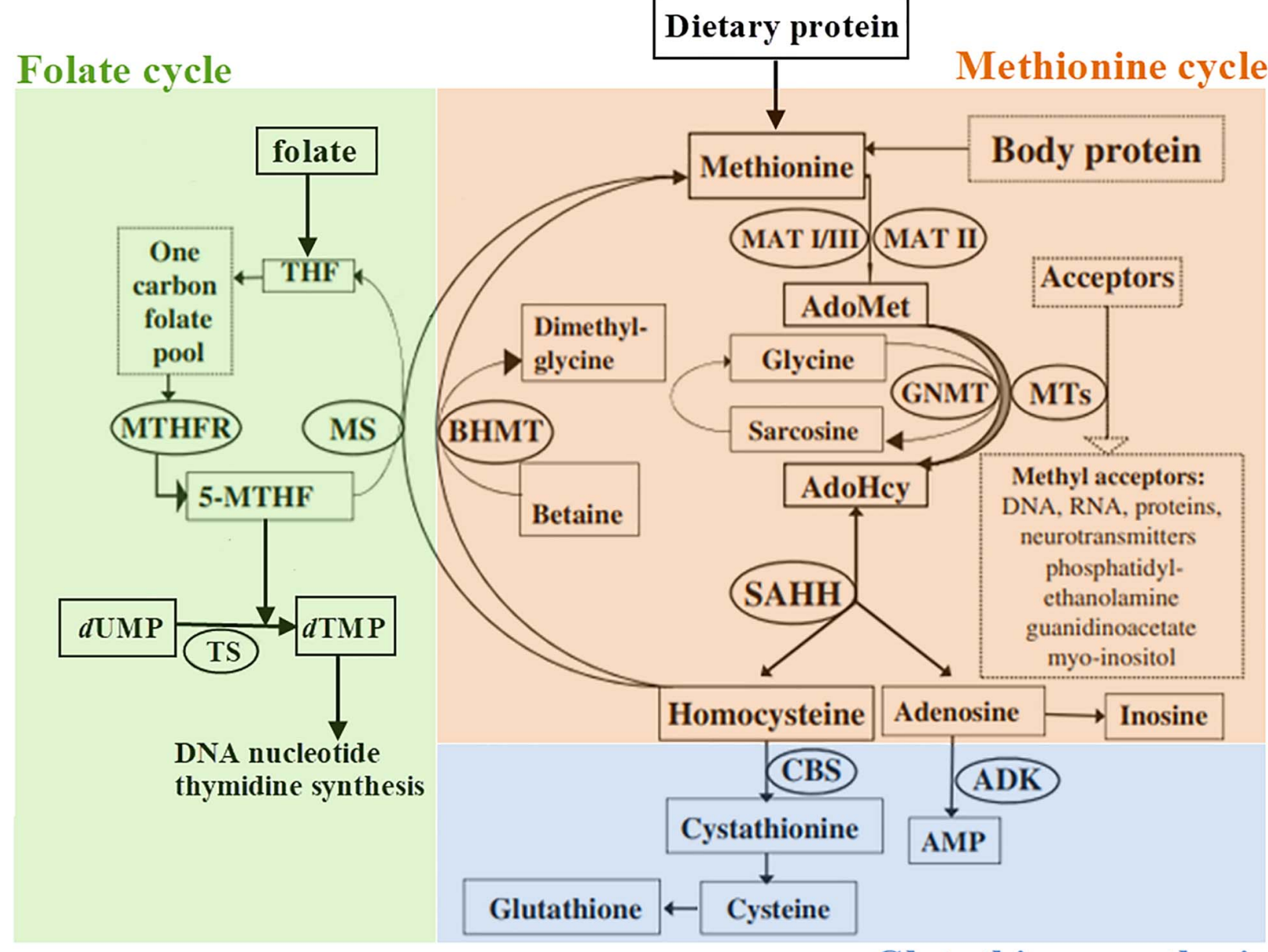

Glutathione synthesis

FIGURE 1 | Schematic representation of the methionine metabolism according to Barić et al. (2017), slightly modified. 5-MTHF, 5-methyltetrahydrofolate; ADK, adenosine kinase; AdoHcy, S-adenosylhomocysteine; AdoMet, S-adenosylmethionine; AMP, adenosine monophosphate; BHMT, betaine-homocysteine methyltransferase; CBS, cystathionine $\beta$-synthase; GNMT, glycine N-methyltransferase; dTMP, $2^{\prime}$-deoxythymidine-5'-monophosphate (thymidylate); dUMP, 2'-deoxyuridine-5'-monophosphate; MAT, methionine adenosyltransferase; MS, methionine synthase; MTHFR, methylenetetrahydrofolate reductase; MTs, a variety of AdoMet-dependent methyltransferases; THF, tetrahydrofolate; SAHH, S-adenosylhomocysteine hydrolase; TS, thymidylate synthase.

There are several examples of enzyme deficiencies in methionine cycle (CBS, SAHHD, MTHFR) with clinical presentation of ASD. It is yet to be elucidated how subtle disturbances in this metabolic pathway affect methylation and consequent epigenetic dysregulation of numerous genes.

Malaguarnera and Cauli (2019) showed the positive clinical effects of L-carnitine administration in non-syndromic forms of autism. Several clinical trials suggest that carnitine supplementation is useful in diminishing symptoms of nonsyndromic ASD. Nevertheless, future clinical trials to identify the subgroup of ASD patients that could benefit from carnitine supplementation are needed (Malaguarnera and Cauli, 2019).

Treating an autistic child is always associated with additional healthcare-associated costs, therapies, education, and family services. The associated social and emotional stresses often lead to depression, somatization, and impairment in quality of life parameters (Glinton and Elsea, 2019).
At the present time, there is not enough evidence for routine supplementation of ASD patients with folic acid, choline, or other supplements. We suggest methionine cycle (Figure 1) to be carefully analyzed in syndromic ASD patients (as proposed earlier, this should be performed by plasma amino acid analysis and total Hcy concentration measurement) and specific supplementation given in case of biochemically proven deficiencies.

\section{CONCLUSION}

Symptoms of ASD can be a present in many IEM, but rarely occur in isolation. Since some of IEM are treatable, physicians treating children with ASD should be aware of a long list of rare and ultra-rare disorders in the differential diagnosis. Given the enormous etiologic variability of autistic symptoms, 
including those of genetic and metabolic origin, diagnostic workup of these patients should include appropriate genetic and metabolic studies elected on the basis of leading symptoms and associated clinical signs. This review is also intended to serve as a reminder on IEM that could present with ASD as a leading or associated presentation. As always, detailed medical history and clinical examination, including detailed neurological examination, should be a basis for planning focused diagnostic work-up of patients with ASD.

\section{AUTHOR CONTRIBUTIONS}

IB conceived the manuscript. TŽ wrote the manuscript. All authors critically revised the manuscript and approved the final version.

\section{REFERENCES}

Abbott, M. H., Folstein, S. E., Abbey, H., and Pyeritz, R. E. (1987). Psychiatric manifestations of homocystinuria due to cystathionine betasynthase deficiency: prevalence, natural history, and relationship to neurologic impairment and vitamin B6-responsiveness. Am. J. Med. Genet. 26, 959-969. doi: 10.1002/ajmg.1320260427

Ahmadabadi, F., Nemati, H., Abdolmohammadzadeh, A., and Ahadi, A. (2020). Autistic feature as a presentation of inborn errors of metabolism. Iran J. Child Neurol. 14, 17-28. doi: 10.22037/ijcn.v414.31672

Asato, M. R., Goldstein, A. C., and Schiff, M. (2015). Autism and inborn errors of metabolism: how much is enough? Dev. Med. Child Neurol. 57, 788-789. doi: $10.111 / \mathrm{dmcn} .12771$

Barić, I., Fumić, K., Glenn, B., Ćuk, M., Schulze, A., Finkelstein, J. D., et al. (2004). S-adenosylhomocysteine hydrolase deficiency in a human: a genetic disorder of methionine metabolism. Proc. Natl. Acad. Sci. U S A 101, 4234-4239. doi: 10.1073/pnas.0400658101

Barić, I., Staufner, C., Augoustides-Savvopoulou, P., Chien, Y. H., Dobbelaere, D., Grünert, S. C., et al. (2017). Consensus recommendations for the diagnosis, treatment and follow-up of inherited methylation disorders. J. Inherit. Metab. Dis. 40, 5-20. doi: 10.1007/s10545-016-9972-7

Baumgartner, M. R., Hörster, F., Dionisi-Vici, C., Haliloglu, G., Karall, D., Chapman, K. A., et al. (2014). Proposed guidelines for the diagnosis and management of methylmalonic and propionic acidemia. Orphanet J. Rare Dis. 9:130. doi: 10.1186/s13023-014-0130-8

Bell, S., Kolobova, I., Crapper, L., and Ernst, C. (2016). Lesch-Nyhan syndrome: models, theories, and therapies. Mol. Syndromol. 7, 302-311. doi: 10.1159/ 000449296

Blau, N., Bélanger-Quintana, A., Demirkol, M., Feillet, F., Giovannini, M., MacDonald, A., et al. (2010). Management of phenylketonuria in Europe: survey results from 19 countries. Mol. Genet. Metab. 99, 109-115. doi: 10.1016/ j.ymgme.2009.09.005

Bublil, E. M., and Majtan, T. (2020). Classical homocystinuria: from cystathionine beta-synthase deficiency to novel enzyme therapies. Biochimie 173, 48-56. doi: 10.1016/j.biochi.2019.12.007

Burrage, L. C., Nagamani, S. C., Campeau, P. M., and Lee, B. H. (2014). Branched-chain amino acid metabolism: from rare Mendelian diseases to more common disorders. Hum. Mol. Genet. 23, 1-8. doi: 10.1093/hmg/ddu 123

Bustad, H. J., Toska, K., Schmitt, C., Vorland, M., Skjærven, L., Kallio, J. P., et al. (2020). A pharmacological chaperone therapy for acute intermittent porphyria. Mol. Ther. 28, 677-689. doi: 10.1016/j.ymthe.2019. 11.010

Campistol, J., Díez-Juan, M., Callejón, L., Fernandez-De Miguel, A., Casado, M., García Cazorla, A., et al. (2016). Inborn error metabolic screening in individuals with nonsyndromic autism spectrum disorders. Dev. Med. Child Neurol. 58, 842-847. doi: 10.1111/dmcn.13114

\section{FUNDING}

The authors gratefully acknowledge the support of the Croatian Science Foundation (HRZZ IP-2019-04-3584) and the Scientific Centre of Excellence for Basic, Clinical and Translational Neuroscience CORE-NEURO ("Experimental and clinical research of hypoxic-ischemic damage in perinatal and adult brain"; GA KK01.1.1.01.0007 funded by the European Union through the European Regional Development Fund).

\section{ACKNOWLEDGMENTS}

TŽ, DPR, and IB are members of the European Reference Network for Rare Hereditary Metabolic Disorders (MetabERN) - Project ID No 739543.

Castro, K., da Silveira Klein, L., Baronio, D., Gottfried, C., Riesgo, R., and Perry, I. S. (2016). Folic acid and autism: what do we know? Nutr. Neurosci. 19, 310-317. doi: 10.1179/1476830514Y.0000000142

Celestino-Soper, P. B. S., Violante, S., Crawford, E. L., Luo, R., Lionel, A. C., Delaby, E., et al. (2012). A common X-linked inborn error of carnitine biosynthesis may be a risk factor for nondysmorphic autism. Proc. Natl. Acad. Sci. U S A 109, 7974-7981. doi: 10.1073/pnas.1120210109

Ciardo, F., Salerno, C., and Curatolo, P. (2001). Neurologic aspects of adenylosuccinate lyase deficiency. J. Child Neurol. 16, 301-308. doi: 10.1177/ 088307380101600501

Connolly, B. S., Feigenbaum, A. S., Robinson, B. H., Dipchand, A. I., Simon, D. K., Tarnopolsky, M. A., et al. (2010). MELAS syndrome, cardiomyopathy, rhabdomyolysis, and autism associated with the A3260G mitochondrial DNA mutation. Biochem. Biophys. Res. Commun. 402, 443-447. doi: 10.1016/j.bbrc. 2010.10.060

Dean, L. (2012). "Methylenetetrahydrofolate reductase deficiency," in Medical Genetics Summaries, eds V. M. Pratt, S. A. Scott, M. Pirmohamed, B. Esquivel, M. S. Kane, B. L. Kattman, et al. (Bethesda, MD: National Center for Biotechnology Information).

Di Meo, I., and Tiranti, V. (2018). Classification and molecular pathogenesis of NBIA syndromes. Eur. J. Paediatr. Neurol. 22, 272-284. doi: 10.1016/j.ejpn. 2018.01.008

Didiášová, M., Banning, A., Brennenstuhl, H., Jung-Klawitter, S., Cinquemani, C., Opladen, T., et al. (2020). Succinic semialdehyde dehydrogenase deficiency: an update. Cells 9:477. doi: 10.20944/preprints202001.0315.v1

Donoghue, S. E., Pitt, J. J., Boneh, A., and White, S. M. (2018). Smith-lemli-opitz syndrome: clinical and biochemical correlates. J. Pediatr. Endocrinol. Metab. 31, 451-459. doi: 10.1515/jpem-2017-0501

Duell, P. B., Salen, G., Eichler, F. S., DeBarber, A. E., Connor, S. L., Casaday, L., et al. (2018). Diagnosis, treatment, and clinical outcomes in 43 cases with cerebrotendinous xanthomatosis. J. Clin. Lipidol. 12, 1169-1178. doi: 10.1016/j. jacl.2018.06.008

El-Hattab, A. W., Adesina, A. M., Jones, J., and Scaglia, F. (2015). MELAS syndrome: clinical manifestations, pathogenesis, and treatment options. Mol. Genet. Metab. 116, 4-12. doi: 10.1016/j.ymgme.2015.06.004

Farr, C. V., El-Kasaby, A., Freissmuth, M., and Sucic, S. (2020). The creatine transporter unfolded: a knotty premise in the cerebral creatine deficiency syndrome. Front. Synaptic Neurosci. 12:588954. doi: 10.3389/fnsyn.2020. 588954

Ferenci, P. (2017). Diagnosis of Wilson disease. Handb. Clin. Neurol. 142, 171-180. doi: 10.1016/B978-0-444-63625-6.00014-8

Fraser, J. L., and Venditti, C. P. (2016). Methylmalonic and propionic acidemias: clinical management update. Curr. Opin. Pediatr. 28, 682-693. doi: 10.1097/ MOP.0000000000000422

Frye, R. E. (2015). Metabolic and mitochondrial disorders associated with epilepsy in children with autism spectrum disorder. Epilepsy Behav. 47, 147-157. doi: 10.1016/j.yebeh.2014.08.134 
Frye, R. E., and Rossignol, D. A. (2011). Mitochondrial dysfunction can connect the diverse medical symptoms associated with autism spectrum disorders. Pediatr. Res. 69, 41-47. doi: 10.1203/PDR.0b013e318212f16b

García-Cazorla, A., Oyarzabal, A., Fort, J., Robles, C., Castejón, E., Ruiz-Sala, P., et al. (2014). Two novel mutations in the BCKDK (branched-chain keto-acid dehydrogenase kinase) gene are responsible for a neurobehavioral deficit in two pediatric unrelated patients. Hum Mutat. 35, 470-477. doi: 10.1002/humu. 22513

Geberhiwot, T., Moro, A., Dardis, A., Ramaswami, U., Sirrs, S., Marfa, M. P., et al. (2018). Consensus clinical management guidelines for niemann-pick disease type C. Orphanet J. Rare Dis. 13:50. doi: 10.1186/s13023-018$0785-7$

Ghaziuddin, M., and Al-Owain, M. (2013). Autism spectrum disorders and inborn errors of metabolism: an update. Pediatr. Neurol. 49, 232-236. doi: 10.1016/j. pediatrneurol.2013.05.013

Glinton, K. E., and Elsea, S. H. (2019). Untargeted metabolomics for autism spectrum disorders: current status and future directions. Front. Psychiatry. 10:647. doi: 10.3389/fpsyt.2019.00647

Hashmi, M. S., and Gupta, V. (2021). "Hartnup disease," in StatPearls [Internet], (Treasure Island, FL: StatPearls Publishing), Available online at: https://www. ncbi.nlm.nih.gov/books/NBK559076 (accessed February 6, 2021).

Hyland, K., Shoffner, J., and Heales, S. J. (2010). Cerebral folate deficiency. J. Inherit. Metab. Dis. 33, 563-570. doi: 10.1007/s10545-010-9159-6

Jaeken, J., and Van den Berghe, G. (1984). An infantile autistic syndrome characterised by the presence of succinylpurines in body fluids. Lancet 2, 1058-1061.

James, S. J., Melnyk, S., Fuchs, G., Reid, T., Jernigan, S., Pavliv, O., et al. (2009). Efficacy of methylcobalamin and folinic acid treatment on glutathione redox status in children with autism. Am. J. Clin. Nutr. 89, 425-430. doi: 10.3945/ ajen.2008.26615

Johnson, T. B., Cain, J. T., White, K. A., Ramirez-Montealegre, D., Pearce, D. A., and Weimer, J. M. (2019). Therapeutic landscape for Batten disease: current treatments and future prospects. Nat. Rev. Neurol. 15, 161-178. doi: 10.1038/ s41582-019-0138-8

Jurecka, A., Zikanova, M., Kmoch, S., and Tylki-Szymańska, A. (2015). Adenylosuccinate lyase deficiency. J. Inherit. Metab. Dis. 38, 231-242. doi: 10.1007/s10545-014-9755-y

Kim, H.-Y. H., Korade, Z., Tallman, K. A., Liu, W., Weaver, C. D., Mirnics, K., et al (2016). Inhibitors of 7-dehydrocholesterol reductase: screening of a collection of pharmacologically active compounds in Neuro2a cells. Chem. Res. Toxicol. 29, 892-900. doi: 10.1021/acs.chemrestox.6b00054

Kiykim, E., Zeybek, C. A., Zubarioglu, T., Cansever, S., Yalcinkaya, C., Soyucen, E., et al. (2016). Inherited metabolic disorders in Turkish patients with autism spectrum disorders. Autism Res. 9, 217-223. doi: 10.1002/aur. 1507

Kruger, W. D. (2017). Cystathionine $\beta$-synthase deficiency: of mice and men. Mol. Genet. Metab. 121, 199-205. doi: 10.1016/j.ymgme.2017.05.011

Li, X., Chauhan, A., Sheikh, A. M., Patil, S., Chauhan, V., Li, X.-M., et al. (2009). Elevated immune response in the brain of autistic patients. J. Neuroimmunol. 207, 111-116. doi: 10.1016/j.neuroim.2008.12.002

Li, X., Li, Y., Li, S., Li, H., Yang, C., and Lin, J. (2020). The role of Shh signalling pathway in central nervous system development and related diseases. Cell Biochem. Funct. 39, 180-189. doi: 10.1002/cbf.3582

Luder, A. S., Mamet, R., Farbstein, I., and Schoenfeld, N. (2009). Awareness is the name of the game: clinical and biochemical evaluation of a case of a girl diagnosed with acute intermittent porphyria associated with autism. Cell. Mol. Biol. 55, 19-22.

Lütjohann, D., Stellard, F., and Björkhem, I. (2020). Levels of 7alphahydroxycholesterol and/or 7alpha-hydroxy-4-cholest-3-one are the optimal biochemical markers for the evaluation of treatment of cerebrotendinous xanthomatosis. J. Neurol. 267, 572-573. doi: 10.1007/s00415-019-09650-0

Madaan, P., Jauhari, P., Luhar, Z. M., Chakrabarty, B., and Gulati, S. (2020). Autism, epilepsy, and neuroregression: photosensitivity on electroencephalography solved the riddle. Clin. EEG Neurosci. 51, 399-402. doi: $10.1177 / 1550059419899327$

Maenner, M. J., Shaw, K. A., Baio, J., Washington, A., Patrick, M., DiRienzo, M., et al. (2020). Prevalence of autism spectrum disorder among children aged 8 years - autism and developmental disabilities monitoring network, 11 sites,
United States, 2016. MMWR Surveill. Summ. 69, 1-12. doi: 10.15585/mmwr. ss6904a1

Malaguarnera, M., and Cauli, O. (2019). Effects of l-carnitine in patients with autism spectrum disorders: review of clinical studies. Molecules 24:4262. doi: $10.3390 /$ molecules 24234262

Malik, M., Sheikh, A. M., Wen, G., Spivack, W., Brown, W. T., and Li, X. (2011). Expression of inflammatory cytokines, Bcl2 and cathepsin D are altered in lymphoblasts of autistic subjects. Immunobiology 216, 80-85. doi: 10.1016/j. imbio.2010.03.001

Márquez-Caraveo, M. E., Ibarra-González, I., Rodríguez-Valentín, R., RamírezGarcía, M. Á, Pérez-Barrón, V., Lazcano-Ponce, E., et al. (2020). Brief report: delayed diagnosis of treatable inborn errors of metabolism in children with autism and other neurodevelopmental disorders. J. Autism Dev. Disord. doi: 10.1007/s10803-020-04682-2 [Epub ahead of Print].

Matsumoto, S., Häberle, J., Kido, J., Mitsubuchi, H., Endo, F., and Nakamura, K. (2019). Urea cycle disorders-update. J. Hum. Genet. 64, 833-847. doi: 10.1038/ s10038-019-0614-4

Mercimek-Andrews, S., and Salomons, G. S. (2015). "Creatine deficiency syndromes", in GeneReviews [Internet], eds M. P. Adam, H. H. Ardinger, R. A. Pagon, et al. (Seattle, WA: University of Washington), 1993-2021.

Morris, A. A., Kožich, V., Santra, S., Andria, G., Ben-Omran, T. I., Chakrapani, A. B., et al. (2017). Guidelines for the diagnosis and management of cystathionine $\beta$-synthase deficiency. J. Inherit. Metab. Dis. 40, 49-74. doi: 10. 1007/s10545-016-9979-0

Mulligan, C., and Bronstein, J. M. (2020). Wilson disease: an overview and approach to management. Neurol. Clin. 38, 417-432. doi: 10.1016/j.ncl.2020. 01.005

Newton, J., Milstien, S., and Spiegel, S. (2018). Niemann-pick type C disease: the atypical sphingolipidosis. Adv. Biol. Regul. 70, 82-88. doi: 10.1016/j.bior.2018. 08.001

Nguyen, K. V. (2019). Beta-amyloid precursor protein (APP) and the human diseases. AIMS Neurosci. 6, 273-281. doi: 10.3934/Neuroscience.2019.4.273

Novarino, G., El-Fishawy, P., Kayserili, H., Meguid, N. A., Scott, E. M., Schroth, J., et al. (2012). Mutations in BCKD-kinase lead to a potentially treatable form of autism with epilepsy. Science 338, 394-397. doi: 10.1126/science.1224631

Orbak, Z., Ertekin, V., Selimoglu, A., Yilmaz, N., Tan, H., Konak, M., et al. (2010). Hartnup disease masked by kwashiorkor. J. Health Popul. Nutr. 28, 413-415. doi: $10.3329 /$ jhpn.v28i4.6049

Orenbuch, A., Fortis, K., Taesuwan, S., Yaffe, R., Caudill, M. A., Golan, H. M., et al. (2019). Prenatal nutritional intervention reduces autistic-like behavior rates among Mthfr-deficient mice. Front. Neurosci. 13:383. doi: 10.3389/fnins. 2019.00383

Patterson, M. C., Garver, W. S., Giugliani, R., Imrie, J., Jahnova, H., Meaney, F. J., et al. (2020). Long-term survival outcomes of patients with Niemann-Pick disease type $\mathrm{C}$ receiving miglustat treatment: a large retrospective observational study. J. Inherit. Metab. Dis. 43, 1060-1069. doi: 10.1002/jimd.12245

Pearl, P. L., Wiwattanadittakul, N., Roullet, J.-B., and Gibson, K. M. (2016). "Succinic semialdehyde dehydrogenase deficiency", in GeneReviews [Internet], eds M. P. Adam, H. H. Ardinger, R. A. Pagon, et al. (Seattle, WA: University of Washington), 1993-2021.

Pillai, N. R., Yubero, D., Shayota, B. J., Oyarzábal, A., Ghosh, R., Sun, Q., et al. (2019). Loss of CLTRN function produces a neuropsychiatric disorder and a biochemical phenotype that mimics Hartnup disease. Am. J. Med. Genet. A 179, 2459-2468. doi: 10.1002/ajmg.a.61357

Polšek, D., Jagatic, T., Cepanec, M., Hof, P. R., and Šimić, G. (2011). Recent developments in neuropathology of autism spectrum disorders. Transl. Neurosci. 2, 256-264. doi: 10.2478/s13380-011-0024-3

Pope, S., Artuch, R., Heales, S., and Rahman, S. (2019). Cerebral folate deficiency: analytical tests and differential diagnosis. J. Inherit. Metab. Dis. 42, 655-672. doi: 10.1002/jimd.12092

Sadigurschi, N., and Golan, H. M. (2019). Maternal and offspring methylenetetrahydrofolate-reductase genotypes interact in a mouse model to induce autism spectrum disorder-like behavior. Genes Brain Behav. 18:e12547. doi: $10.1111 / \mathrm{gbb} .12547$

Samuraki, M., Komai, K., Hasegawa, Y., Kimura, M., Yamaguchi, S., Terada, N., et al. (2008). A successfully treated adult patient with L-2-hydroxyglutaric aciduria. Neurology 70, 1051-1052. doi: 10.1212/01.wnl.0000287141.90944.95 
Šarac, H., Henigsberg, N., Markeljević, J., Pavliša, G., Hof, P. R., and Šimić, G. (2011). Fragile X-premutation tremor/ataxia syndrome (FXTAS) in a young woman: clinical, genetics, MRI and ${ }^{1} \mathrm{H}-\mathrm{MR}$ spectroscopy correlates. Coll. Antropol. 35, 327-332.

Saudubray, J. M., and García-Cazorla, A. (2018). An overview of inborn errors of metabolism affecting the brain: from neurodevelopment to neurodegenerative disorders. Dialogues Clin. Neurosci. 20, 301-325. doi: 10.31887/DCNS.2018.20. 4/jmsaudubray

Schaefer, G. B., Mendelsohn, N. J., and Professional Practice and Guidelines Committee. (2013). Clinical genetics evaluation in identifying the etiology of autism spectrum disorders: 2013 guideline revisions. Genet. Med. 15, 399-407. doi: 10.1038/gim.2013.32

Schiff, M., Benoist, J. F., Aïssaoui, S., Boespflug-Tanguy, O., Mouren, M.-C., de Baulny, H. O., et al. (2011). Should metabolic diseases be systematically screened in nonsyndromic autism spectrum disorders? PLoS One 6:e21932. doi: 10.1371/journal.pone.0021932

Seker Yilmaz, B., Davison, J., Jones, S. A., and Baruteau, J. (2021). Novel therapies for mucopolysaccharidosis type III. J. Inherit. Metab. Dis. 44, 129-147. doi: 10.1002/jimd.12316

Šimić, G., Vukić, V., Kopić, J., Krsnik, Ž, and Hof, P. R. (2020). Molecules, mechanisms, and disorders of self-domestication: keys for understanding emotional and social communication from an evolutionary perspective. Biomolecules 11:2. doi: 10.3390/biom 11010002

Simons, A., Eyskens, F., Glazemakers, I., and van West, D. (2017). Can psychiatric childhood disorders be due to inborn errors of metabolism? Eur. Child Adolesc. Psychiatry 26, 143-154. doi: 10.1007/s00787-016-0908-4

Singer, D., and Camargo, S. M. (2011). Collectrin and ACE2 in renal and intestinal amino acid transport. Channels (Austin) 5, 410-423. doi: 10.4161/chan.5.5. 16470

Spilioti, M., Evangeliou, A. E., Tramma, D., Theodoridou, Z., Metaxas, S., Michailidi, E., et al. (2013). Evidence for treatable inborn errors of metabolism in a cohort of 187 Greek patients with autism spectrum disorder (ASD). Front. Hum. Neurosci. 7:858. doi: 10.3389/fnhum.2013.00858

Spiritos, Z., Salvador, S., Mosquera, D., and Wilder, J. (2019). Acute intermittent porphyria: current perspectives and case presentation. Ther. Clin. Risk Manag. 15, 1443-1451. doi: 10.2147/TCRM.S180161

Strauss, K. A., Ferreira, C., Bottiglieri, T., Zhao, X., Arning, E., Zhang, S., et al. (2015). Liver transplantation for treatment of severe S-adenosylhomocysteine hydrolase deficiency. Mol. Genet. Metab. 116, 44-52. doi: 10.1016/j.ymgme. 2015.06.005

ten Hoedt, A. E., de Sonneville, L. M. J., Francois, B., ter Horst, N. M., Janssen, M. C. H., Rubio-Gozalbo, M. E., et al. (2011). High phenylalanine levels directly affect mood and sustained attention in adults with phenylketonuria: a randomised, double-blind, placebo-controlled, crossover trial. J. Inherit. Metab. Dis. 34, 165-171. doi: 10.1007/s10545-010-9253-9

Thurm, A., Tierney, E., Farmer, C., Albert, P., Joseph, L., Swedo, S., et al. (2016). Development, behavior, and biomarker characterization of Smith-Lemli-Opitz syndrome: an update. J. Neurodev. Disord. 8:12. doi: 10.1186/s11689-0169145-x

Van Schaftingen, E., Rzem, R., and Veiga-da-Cunha, M. L. (2009). L-2Hydroxyglutaric aciduria, a disorder of metabolite repair. J. Inherit. Metab. Dis. 32, 135-142. doi: 10.1007/s10545-008-1042-3

van Wegberg, A. M. J., Evers, R. A. F., van Dam, E., de Vries, M. C., Janssen, M. C. H., Heiner-Fokkema, M. R., et al. (2020). Does the 48-hour BH4 loading test miss responsive PKU patients? Mol. Genet. Metab. 129, 186-192. doi: 10. 1016/j.ymgme.2019.12.011

van Wegberg, A. M. J., MacDonald, A., Ahring, K., Bélanger-Quintana, A., Blau, N., Bosch, A. M., et al. (2017). The complete European guidelines on phenylketonuria: diagnosis and treatment. Orphanet J. Rare Dis. 12:162. doi: 10.1186/s13023-017-0685-2

Veeravigrom, M., Desudchit, T., Chomtho, K., and Pongpunlert, W. (2014). Neurodegeneration with brain iron accumulation disorder mimics autism. Pediatr. Neurol. 51, 862-863. doi: 10.1186/2047-9158-2-17

Wagner, V. F., and Northrup, H. (2019). "Mucopolysaccharidosis type III," in GeneReviews, eds M. P. Adam, H. H. Ardinger, R. A. Pagon, S. E. Wallace, L. J. H. Bean, G. Mirzaa, et al. (Seattle, WA: University of Washington, Seattle).

Waye, M. M. Y., and Cheng, H. Y. (2018). Genetics and epigenetics of autism: a review. Psychiatry Clin. Neurosci. 72, 228-244. doi: 10.1111/pcn.12606

Wijburg, F. A., Węgrzyn, G., Burton, B. K., and Tylki-Szymańska, A. (2013). Mucopolysaccharidosis type III (Sanfilippo syndrome) and misdiagnosis of idiopathic developmental delay, attention deficit/hyperactivity disorder or autism spectrum disorder. Acta Paediatr. 102, 462-470. doi 10.1111/ apa.12169

Yilmaz, K. (2009). Riboflavin treatment in a case with l-2-hydroxyglutaric aciduria. Eur. J. Paediatr. Neurol. 13, 57-60. doi: 10.1016/j.ejpn.2008.01.003

Yoganathan, S., Arunachal, G., Sudhakar, S. V., Rajaraman, V., Thomas, M. and Danda, S. (2016). $\beta$-Propeller protein-associated neurodegeneration: a rare cause of infantile autistic regression and intracranial calcification. Neuropediatrics 47, 123-127. doi: 10.1055/s-0035-1571189

Zafeiriou, D. I, Ververi, A., Salomons, G. S., Vargiami, E., Haas, D., Papadopoulou, V., et al. (2008). L-2-Hydroxyglutaric aciduria presenting with severe autistic features. Brain Dev. 30, 305-307. doi: 10.1016/j.braindev.2007.09.005

Conflict of Interest: The authors declare that the research was conducted in the absence of any commercial or financial relationships that could be construed as a potential conflict of interest.

Copyright (C) 2021 Žigman, Petković Ramadža, Šimić and Barić. This is an openaccess article distributed under the terms of the Creative Commons Attribution License (CC BY). The use, distribution or reproduction in other forums is permitted, provided the original author(s) and the copyright owner(s) are credited and that the original publication in this journal is cited, in accordance with accepted academic practice. No use, distribution or reproduction is permitted which does not comply with these terms. 